Physics Vol. 2, No. 3, pp. 131-139, 1965. Physics Publishing Co. Printed in Great Britain

\title{
THE BOHR-SOMMERFELD QUANTIZATION RULE AND THE WEYL CORRESPONDENCE
}

\author{
P.N. ARGYRES \\ Lincoln Laboratory, ${ }^{*}$ Massachusetts Institute \\ of Technology Lexington, Massachusetts 021:3 \\ (Received 17 May 1965 )
}

\begin{abstract}
With the use of the Weyl correspondence between quantum mechanical operators and classical dynamical functions, an exact quantization rule is derived for a system with one degree of freedom and arbitrary Hamiltonian. In the semiclassical limit of small Planck's constant, this reduces to the Bohr-Sommerfeld quantization rule as derived by the WKB approximation. Higher order terms are also derived. It is further proven that for a simple harmonic oscillator the Bohr-Sommerfeld rule must give the exact energy eigenvalues for all states.
\end{abstract}

\section{Introduction}

AN APPROXIMATE RULE for the quantization of the energy of a periodic system with one degree of freedom is given by the well-known Bohr-Sommerfeld (B.-S.) quantization rule, which states that the action integral of the system must equal a half-integer times Planck's constant. In this form, which is slightly modified from the original one postulated by Bohr, this rule has been derived [1] on the basis of the WKB (semi-classical, or asymptotic) approximation to the Schrodinger equation for the wave function of the system. This is an asymptotic approximation valid only for small values of Planck's constant. Only the simplest system with a Hamiltonian of the form $H(p, q)=\left(p^{2} / 2 m\right)+V(q)$ has been considered. The derivation depends crucially on on the so-called "connection formulas" for the wave function on either side of-the classical turning points. The establishment of the connection formulas is a rather delicate mathematical problem and it has been considered in detail by many authors [2-6]. The B. -S. quantization rule has thus been proven for the simple system to hold true only for large quantum numbers. No correction terms to the quantization rule have been derived on the basis of the WKB approximation, as far as we are aware. In this connection the simple harmonic oscillator has presented a curious case, in that its energy eigenvalues are given exactly by the B.-S. quantization rule for all quantum numbers. This has been considered as an accident $[1,7]$.

In the following we present a quantization rule which is exact and its meaning and origin obvious. This is expressed in terms of the quantum mechanical operators of the system and it

* Operated with support from the U.S. Air Force. 
is shown that it can be written in terms of an integral over the classical phase space of the system. It is proven that this quantization rule is valid for a system of one degree of freedom but with an arbitrary Hamiltonian, provided its energy spectrum is non-degenerate. In this proof use is made of the Weyl correspondence [8] between quantum mechanical operators and classical dynamical functions. This generalization allows the application of this quantization rule to effective one-dimensional problems usually arising from separable three-dimensional ones.

It is further shown that to the lowest order in $\hbar$ ( $=$ Planck' $s$ constant divided by $2 \pi$ ) this exact relation gives the B.-S. quantization rule, which is thus derived without the use of any wave functions or connection formulas. In addition, a procedure is developed which yields corrections to the B. -S. rule in power series in $\hbar^{2}$, and the first correction is given explicitly. This may provide a quantitative measure of the inaccuracy of energy eigenvalues as given by the B. - S. rule.

The case of a harmonic oscillator is considered in detail. It is shown that the corrections to the B. -S. action integral vanish for this system to all orders in $\hbar$, which explains the exactness of the energy eigenvalues as obtained from the B. -S. rule for this system.

\section{Exact quantization rule}

Let us consider a system of one degree of freedom with the classical Hamiltonian $H(p, q)=$ $\left(p^{2} / 2 m\right)+V(q)$, where $q$ is the Cartesian coordinate ranging from - $\infty$ to $\infty$ and $p$ the conjugate momentum. We take $V(q)$ to be such that the classical motion is periodic, at least for a certain range of values of its total energy. Quantum mechanically the energies of the stationary states of this system are given by the eigenvalues $\varepsilon_{n}$ of the Hamiltonian operator $\mathscr{H}=H(P, Q)=$ $\left(P^{2} / 2 m\right)+V(O)$, where $P$ and $Q$ are the momentum and position operators, respectively, satisfying the commutation relation $[Q, P]=i \hbar$.

For the discrete spectrum we know that the energy eigenvalues are non-degenerate. We can then enumerate the bound states with energies $\varepsilon_{n}$ by use of the integers $n=0,1,2,3, \ldots$. With the help of the unit step function $s(\varepsilon)$,

$$
s(\varepsilon)= \begin{cases}1, & \varepsilon>0 \\ 1 / 2, & \varepsilon=0 \\ 0, & \varepsilon<0\end{cases}
$$

we have that $\operatorname{tr} s(\varepsilon-\mathscr{H})=\sum_{n}, s\left(\varepsilon-\varepsilon_{n}^{\prime}\right)$ is equal to an integer that gives the number of bound states with eigenenergies less than $\varepsilon$, if $\varepsilon \neq \varepsilon_{n}^{\prime}$. If $\varepsilon$ is equal to one of the eigenvalues, say $\varepsilon_{n}$, we have from $(2.1)$

$$
\operatorname{tr} s\left(\varepsilon_{n}-\mathscr{H}\right)=n+1 / 2
$$

where $n=0,1,2,3, \ldots$. Clearly, an independent evaluation of the trace in equation (2.2) will yield an equation for the energy eigenvalues $\varepsilon_{n}$ of the system.

It is possible now to express the trace in a form of an integral over the classical phase space of the system. Furthermore, this form lends itself easily to an evaluation in powers of $\hbar^{2}$. This transformation is essentially the same used by Wigner [9] in the study of quantum 
corrections for thermodynamic equilibrium properties by the introduction of the Wigner distribution function. Generalizing slightly Wigner's procedure, we define a classical function $S(p q ; \varepsilon)$ corresponding to the operator $s(\varepsilon-\mathscr{H})$ by the relation

$$
S(p q ; \varepsilon)=\int_{-\infty}^{+\infty} d x \mathrm{e}^{i p x / \hbar}<q-1 / 2 x|s(\varepsilon-\mathscr{H})| q+1 / 2 x>,
$$

where $Q|q>=q| q>$. Now, as Wigner noted, the integrand of equation (2.3) can be written as $\left\langle q-1 / 2 x\left|U^{-1} s U\right| q+1 / 2 x\right\rangle$, where $U=\exp (i Q p / \hbar)$. Furthermore, we have $U^{-1} s(\varepsilon-\mathscr{H}) U=s\left(\varepsilon-U^{-1} \mathscr{H} U\right) \quad$ w1th

$$
U^{-1} \mathscr{H} U=H\left(U^{-1} P U, Q\right)=H(p+P, Q) \text {. }
$$

The last equality is a result of $[P, U]=p U$, which is a direct consequence of the fundamental commutation relation $[Q, P]=i \hbar$. Thus, we can express $(2.3)$ in the form

$$
S(p q ; \varepsilon)=\int_{-\infty}^{+\infty} d x<q-1 / 2 x|s(\varepsilon-H(p+P, Q))| q+1 / 2 x>.
$$

From the definition (2.3) of $S(p q ; \varepsilon)$ it follows that

$$
\operatorname{tr} s(\varepsilon-\mathscr{H})=\frac{1}{2 \pi \hbar} \int_{-\infty}^{+\infty} \int_{-\infty}^{+\infty} d p d q S(p q ; \varepsilon)
$$

as it can easily be seen by carrying out the integration over $p$ first. Equation (2.2) becomes then the exact quantization rule

$$
\frac{1}{2 \pi \hbar} \int_{-\infty}^{+\infty} \int_{-\infty}^{+\infty} d p d q S\left(p q ; \varepsilon_{n}\right)=n+1 / 2
$$

where $S(p q ; \varepsilon)$ is given by $(2.5)$ and $n=0,1,2,3, \ldots$.

It is easy to see that this quantization rule, equations (2.7) and (2.5), is valid for an arbitrary Hamiltonian $\mathscr{H}=H(P, Q)$ provided its energy spectrum is non-degenerate, since, under this condition, none of the steps (2.2) to (2.7) depends on the particular form of the Hamiltonian.

For later reference we must note that in this case the quantum mechanical Hamiltonian function $H(P, Q)$ is not, in general, the same as the corresponding classical Hamiltonian function, which we denote now by $H_{c}(p, q)$, but it is related to it by the Weyl correspondence [8]. This correspondence between any classical dynamical quantity $A_{c}(p, q)$ and the corresponding quantum mechanical operator $A(P, Q)$, to be designated by $A(P, Q) \leftrightarrow A_{c}(p, q)$, is defined as follows: if

then

$$
A_{c}(p, q)=\int_{-\infty}^{+\infty} \int_{-\infty}^{+\infty} d \sigma d \tau a(\sigma, \tau) \mathrm{e}^{i(\sigma p+\tau q)}
$$

$$
A(P, Q)=\int_{-\infty}^{+\infty} \int_{-\infty}^{+\infty} d \sigma d \tau a(\sigma, \tau) \mathrm{e}^{i(\sigma P+\tau Q)}
$$


and vice versa. $a(\sigma, \tau)$ can thus be expressed either in terms of $A_{c}(p, q)$, or in terms of $A(P, Q)$, namely

$$
\begin{aligned}
a(\sigma, \tau) & =\frac{1}{(2 \pi)^{2}} \iint d p d q A_{c}(p, q) \mathrm{e}^{-i(\sigma p+\tau q)} \\
& =\frac{\hbar}{2 \pi} \operatorname{tr}\left\{A(P, Q) \mathrm{e}^{-i(\sigma P+\tau Q)}\right\}
\end{aligned}
$$

since the operators $(\hbar / 2 \pi)^{1 / 2} \exp \{i(\sigma P+\tau Q)\}$ form an orthonormal set $[10,11]$.

With the help of equations (2.8) and (2.9) it is easily shown [10-12] that the relationship between an operator and a classical function as prescribed by equation (2.3) is identical to the Weyl correspondence; for the convenience of the reader, a direct and simple proof of this is presented in the Appendix. Thus we have

$$
S(p q ; \varepsilon) \longleftrightarrow s(\varepsilon-H(P, Q))
$$

We note in particular that the important relation (2.6) is now obvious, as it is obtained from equation (2.9) by putting $\sigma=\tau=0$.

\section{The Bohr-Sommerfeld quantization rule and corrections}

We now prove that to the lowest order in $\hbar$ the integral of the left-hand side of equation (2.7) for a fixed $\varepsilon_{n}$ is just the action integral, and thus equation (2.7) reduces to the B. $-S$. quantization rule. We note that, since in the Schrodinger representation $Q|q>=q| q>$ and $P|q>=i \hbar(\partial / \partial q)| q>$, in the lowest order in $\hbar$ equation (2.5) for $S(p q ; \varepsilon)$ with a fixed parameter $\varepsilon$ becomes equal to

$$
\begin{aligned}
S_{0}(p q ; \varepsilon) & =\int_{-\infty}^{+\infty} d x<q-1 / 2 x\left|s\left(\varepsilon-H_{c}(p, q+1 / 2 x)\right)\right| q+1 / 2 x> \\
& =s\left(\varepsilon-H_{c}(p q)\right) .
\end{aligned}
$$

Thus, from the definition of $s(\varepsilon)$, equation (2.1), and the exact quantization rule (2.7) we get for small $\hbar$ the quantization condition

$$
\frac{1}{2 \pi \hbar} \int_{H_{c} \leqslant \varepsilon_{n}} d p d q=n+1 / 2 .
$$

In the usual case of $H_{c}(p, q)=p^{2} / 2 m+V(q)$ with a potential such that there are two turning points, $a(\varepsilon)$ and $b(\varepsilon)$, for the classical motion of total energy $\varepsilon$, equation (3.2) becomes

$$
(1 / \hbar) \oint p\left(\varepsilon_{n} q\right) d q=(2 / \hbar) \int_{a\left(\varepsilon_{n}\right)}^{b\left(\varepsilon_{n}\right)}\left|p\left(\varepsilon_{n} q\right)\right| d q=(n+1 / 2) 2 \pi \text {, }
$$


where $p(\varepsilon q)$ is the solution of $H_{c}(p, q)=\varepsilon$. This is the B. -S. quantization rule, as it is derived on the basis of the WKB approximation for the Schrodinger equation [1].

One can find correction terms to $S(p q ; \varepsilon)$ for small $\hbar$ and fixed $\varepsilon$ from the exact relation (2.5). For example, an expansion of $s(\varepsilon-H(p+P, Q))$ in powers of $P$ would yield a series expansion of $S(p q)$ in $\hbar$. For such an expansion it is useful to note that $s(\varepsilon)$, as defined by (2.1), has the Fourier transform representation

$$
s(\varepsilon)=\lim _{\eta \rightarrow 0^{+}} \frac{i}{2 \pi} \int_{-\infty}^{+\infty} \frac{d z}{z+i \eta} \mathrm{e}^{-i z \varepsilon}
$$

However, a direct expansion of exp $\{i z[H(p+P, Q)-\varepsilon]\}$ in powers of $P$ is tedious, especially for an arbitrary Hamiltonian. It is more convenient to proceed as follows. According to $(3.4)$ and $(2.10)$ we can write

$$
S(p q)=\lim _{\eta \rightarrow 0^{+}} \frac{i}{2 \pi} \int_{-\infty}^{+\infty} \frac{d z}{z+i \eta} E(p q ; z) \text {. }
$$

where

$$
E(p q ; z) \leftrightarrow \mathscr{E}(P Q ; z) \equiv \operatorname{exo}\{i z[H(P, Q)-\varepsilon]\}
$$

$\leftrightarrow$ indicating as before the Weyl correspondence (2.8). We can now conveniently expand $E(p q ; z)$ in powers of $\hbar$ by constructing an equation for it, which is obtained from the obvious equation for $\mathscr{E}(P Q ; z)$

$$
\frac{1}{i} \frac{\partial}{\partial z} \mathscr{E}(z)=\bar{E} \overline{\mathscr{H}}=\frac{1}{2}(\mathscr{E} \overline{\mathscr{H}}+\overline{\mathscr{H}} \mathscr{E})
$$

where we have put $\overline{\mathscr{H}} \equiv \mathscr{H}-\varepsilon=H(P, Q)-\varepsilon$. The Weyl correspondence (3.6) allows us to write immediately the corresponding equation for $E(z)$ in terms of $\bar{H}_{c}(p, q) \equiv H_{c}(p, q)-\varepsilon \leftrightarrow \overline{\mathscr{H}}$, if we make use of the expression, derived by Groenewold [10], for the classical quantity corresponding to $\frac{1}{2}(\mathscr{E} \overline{\mathscr{H}}+\overline{\mathscr{H}} \mathscr{E})$. We have

$$
\frac{1}{i} \frac{\partial}{\partial z} E(p q ; z)=\bar{H}_{c}(p, q)\left\{\cos \frac{\hbar}{2}\left(\frac{\delta}{\delta p} \frac{\partial}{\partial q}-\frac{\delta}{\delta q} \frac{\partial}{\partial p}\right)\right\} E(p q ; z)
$$

with $E(z=0)=1$. Here $\delta / \delta p, \delta / \delta q$ operate only on the function to their left, whereas $\partial / \partial p, \partial / \partial q$ operate only on the function to their right. We can now find a solution of in power series in $\hbar^{2}$,

$$
E(z)=\mathrm{e}^{i z \bar{H}_{c}} \sum_{r=0}^{\infty} \hbar^{2} r_{r}(z)
$$

since from the expansion of the cosine in (3.8) it is obvious that the coefficients of the odd powers of $\hbar$ vanish. From (3.8) we thus find $G_{0}(z)=1$ and 


$$
G_{1}(z)=\frac{1}{8}\left(\frac{z^{2}}{2} H_{c}\left(\frac{\delta}{\delta p} \frac{\partial}{\partial q}-\frac{\delta}{\delta q} \frac{\partial}{\partial p}\right)^{2} H_{c}+\frac{i z^{3}}{3} H_{c}\left(\frac{\delta}{\delta p} \frac{\partial H_{c}}{\partial q}-\frac{\delta}{\delta q} \frac{\partial H_{c}}{\partial p}\right)^{2}\right\}
$$

By substituting equations (3.10) and (3.9) in equation (3.5), we obtain

$$
S(p q)=S_{0}(p q)+\hbar^{2} S_{1}(p q)+0\left(\hbar^{4}\right)
$$

where $S_{0}(p q ; \varepsilon)=s\left(\varepsilon-H_{c}(p q)\right)$, in accordance with (3.1), and

$$
\begin{aligned}
S_{1}(p q ; \varepsilon) & =\frac{1}{16}\left\{H_{c}\left(\frac{\delta}{\delta p} \frac{\partial}{\partial q}-\frac{\delta}{\delta q} \frac{\partial}{\partial p}\right)^{2} H_{c}\right\} \delta^{\prime}\left(H_{c}-\varepsilon\right) \\
& +\frac{1}{24} H_{c}\left(\frac{\delta}{\delta p} \frac{\partial H_{c}}{\partial q}-\frac{\delta}{\delta q} \frac{\partial H_{c}}{\partial p}\right)^{2} \delta^{\prime \prime}\left(H_{c}-\varepsilon\right) .
\end{aligned}
$$

In arriving at (3.12) we have made use of $s^{\prime}(\varepsilon) \equiv d s / d \varepsilon=\delta(\varepsilon)$, the Dirac delta function. Thus, the B. $-S$. quantization rule (3.2) gives good results for those values of $\varepsilon_{n}$ for which

$$
\hbar^{2} \iint d p d q S_{1}\left(p q ; \varepsilon_{n}\right) \ll \iiint_{H_{c}} \leqslant \varepsilon_{n} d p d q .
$$

For the simple case $H_{c}=\left(p^{2} / 2 m\right)+V(q)$ of common interest, we get from (3.12)

$S_{1}(p q ; \varepsilon)=\frac{1}{24 m}\left\{3 V^{\prime \prime}(q) \delta^{\prime}\left(H_{c}-\varepsilon\right)+\left[V^{\prime}(q)^{2}+\frac{p^{2}}{m} V^{\prime \prime}(q)\right] \delta^{\prime \prime}\left(H_{c}-\varepsilon\right)\right\}$.

The phase space integral of $S_{1}$ as given by (3.14) can be carried out by a change to the variables $y=H_{c}(p, q)$ and $q$, the Jacobian of the transformation being $m /|p(y q)|=$ $m / \sqrt{2 m[y-V(q)]}$. If we denote the turning points of the classical motion of energy $\varepsilon$ by $a(\varepsilon)$ and $b(\varepsilon)$, then we find for this case by integration by parts

$$
\hbar^{2} \iint d p d q S_{1}(p q ; \varepsilon)=-\frac{\hbar^{2}}{12} \frac{d}{d \varepsilon} \int_{a(\varepsilon)}^{b(\varepsilon)} \frac{V^{\prime \prime}(q)}{p(\varepsilon q)} d q
$$

provided $V^{\prime}(a), V^{\prime}(b)$ are finite and non-vanishing and $V^{\prime \prime}(a), V^{\prime \prime}(b)$ are finite.

\section{The simple harmonic oscillator}

The case of a simple harmonic oscillator is of interest here, because the approximate B. -S. quantization rule (3.3) gives all the energy eigenvalues exactly. In the context of the WKB approximation this has to be considered an accident [1,7]. It is interesting, therefore, to inquire whether the present treatment allows us to justify this circumstance. We show below that indeed the exact quantization rule, equation (2.7), reduces for this case to the B. $-S$ rule $(3.3)$; i.e. we prove that for the harmonic oscillator 


$$
\iint d p d q S(p q ; \varepsilon)=\oint p(\varepsilon q) d q
$$

to all orders in $\hbar$.

We first note that on account of the special form of the Hamiltonian for this case $H(p, q)=$ $p^{2}+q^{2}$ (dropping irrelevant constants), equation $(3.8)$ for $E(p q ; z)$ becomes simply

$$
\frac{1}{i} \frac{\partial E}{\partial z}=\left[H-\varepsilon-\frac{\hbar^{2}}{4}\left(\frac{\partial^{2}}{\partial p^{2}}+\frac{\partial^{2}}{\partial q^{2}}\right)\right] E,
$$

with $E(p q ; 0)=1$. We can prove further that $E(p q, z)$ for this case depends on $p$ and $q$ only through the combination $H(p, q)$ i.e. $E=E(H, z)$. To see this, we note from the definition of $\mathscr{E}(z)$, equation $(3.6)$, that $[\mathscr{E}(z), \overline{\mathscr{H}}]=0$, where $[$,$] denotes the commutator. Now, as$ Groenewold [10] has shown,

$$
\frac{i}{2}[\mathscr{E}, \overline{\mathscr{H}}] \leftrightarrow \bar{H}(p, q)\left\{\sin \frac{\hbar}{2}\left(\frac{\delta}{\delta p} \frac{\partial}{\partial q}-\frac{\delta}{\delta q} \frac{\partial}{\partial p}\right)\right\} E(p q) .
$$

For the harmonic oscillator we thus have simply

$$
p \frac{\partial E}{\partial q}-q \frac{\partial E}{\partial p}=0
$$

the general solution of which is that $E(p q)$ depends on $p$ and $q$ only through $H(p, q)=p^{2}+q^{2}$. This is consistent with (4.2), since it can easily be checked that for such functions $E(H(p q), z)$ equation (4.2) takes the form

$$
\frac{1}{i} \frac{\partial E(H, z)}{\partial z}=\left[H-\varepsilon-\hbar^{2}\left(H \frac{\partial^{2}}{\partial H^{2}}+\frac{\partial}{\partial H}\right)\right] E(H, z) .
$$

Writing $E(H, z)=\exp [i z(H-\varepsilon)] G(H, z)$ we find from (4.5) that

$$
\frac{1}{i} \frac{\partial}{\partial z} G(H, z)=-\hbar^{2} \mathrm{e}^{-i z H}\left(H \frac{\partial^{2}}{\partial H^{2}}+\frac{\partial}{\partial H}\right) \mathrm{e}^{i z H} G(H, z)
$$

with $G(H, 0)=1$, or equivalently

$$
G(H, z)=1-i \hbar^{2} \int_{0}^{z} d y K\left(H, \frac{\partial}{\partial H^{\prime}} y\right) G(H, y)
$$

with

$$
K\left(H, \frac{\partial}{\partial H^{\prime}} y\right)=H\left(\frac{\partial}{\partial H}+i y\right)^{2}+\frac{\partial}{\partial H}+i y
$$

Iterating equation (4.7) we obtain the solution $G(H, z)$ in power series of $\hbar^{2}: G=\sum_{r=0}^{\infty} \hbar^{2} r_{r}$ 
with $G_{0}=1$ and

$$
G_{r}(H, z)=-i \hbar^{2} \int_{0}^{z} d y K\left(H, \frac{\partial}{\partial H^{\prime}} y\right) G_{r-1}(H, y)
$$

The corresponding expansion of $E=\exp (i z \bar{H}) G(H, z)$ gives through equation (3.5) a power series expansion of $S(p q ; \varepsilon)=\sum_{r=0}^{\infty} \hbar^{2}{ }^{r} S_{r}(H, \varepsilon)$. Clearly the term $G_{0}=1$ gives $S_{0}=s(\varepsilon-H)$, the integral of which over the phase space is exactly the right-hand side of equation (4.1). We shall now prove that all the remaining terms $S_{r}(r \geqslant 1)$ have vanishing phase-space integrals, i.e.

$$
\iint d p d q S_{r}(H(p, q), \varepsilon)=\pi \int_{0}^{\infty} d H S_{r}(H, \varepsilon)=0(r \geqslant 1)
$$

This will then complete the proof of the desired equation (4.1). In order to establish (4.10), we note that for $r=1$ equation (4.9) gives $G_{1}=-i \hbar^{2}\left[\left(i z^{2} / 2\right)-\left(z^{3} / 3\right) H\right]$, as it can be seen from equation (3.10) for this case. Now it is easily checked that, on account of the special form of $K(H, \partial / \partial H, y)$, successive application of equation (4.9) gives for $G_{r}(H, z)$ $(r \geqslant 1)$ a sum of terms of the form $H^{\alpha} z^{\alpha+1+\beta}$, where $\alpha$ and $\beta$ are integers and $\beta \geqslant 1$. Thus, sinces $(\alpha+1)(\varepsilon)=\delta^{(\alpha)}(\varepsilon)$, where the superscript $(\alpha)$ denotes the $\alpha$-th derivative, equation (3.5) gives for each $S_{r}(H, \varepsilon)$ with $r \geqslant 1$ a sum of terms of the form $H^{\alpha} \delta^{(\alpha+\beta)}(H-\varepsilon)$. Thus, since $\beta \geqslant 1$, the integral over $H$ of all $S_{r}$ with $r \geqslant 1$ vanishes, which proves equation (4.10) and completes the proof of the desired equation (4.1).

\section{Acknowledgement}

It is a pleasure to acknowledge an interesting conversation on this topic with Prof. L.M. Roth.

\section{References}

1. See any book on Quantum Mechanics, e.g. A. MESSIAH, Quantum Mechanics, Vol. 1, NorthHolland Publishing Co., Amsterdam (1961).

2. E.C. KEMBLE, Phys. Rev. 48, 549 (1935)

3. R.E. LANGER, Phys. Rev. 51, 669 (1937).

4. E.C. KEMBLE, Fundamental Principles of Quantum Mechanics, pp. 90-112, McGraw-Hill, New York (1937).

5. W. H. FURRY, Phys. Rev. 71, 360 (1946).

6. E.C. TITChMARCH, J. Math. Oxford 5, 228 (1954).

7. See, however, reference 4 for a discussion of this case.

8. H. WEYL, Z. Phys, 46, 1 (1927).

9. E. WIGNER, Phys. Rev. 40, 749 (1932). 
10. H. J. GROENEWOLD, Physica 12, 405 (1946).

11. J.E. MOYAL, Proc. Camr. Phil. Soc. 45, 99 (1949).

12. K. SCHRAM and B.R.A. NIJBOER, Physica 25, 733 (19591.

\section{Appendix}

We prove here the identity of the Weyl correspondence between classical and quantum mechanical dynamical quantities, equations (2.8), to that given by equation (2.3), i.e. if $A(P, Q)$ and $A_{c}(p, q)$ are related by $(2.8)$, then

$$
A_{c}(p, q)=\int_{-\infty}^{+\infty} d x \mathrm{e}^{i p x / \hbar}<q-1 / 2 x|A(P, Q)| q+1 / 2 x>
$$

We first recall that for any two operators $B$ and $C$ such that their commutator $[B, C]$ commutes with both of them we have the identity

$$
\mathrm{e}^{B+C}=\mathrm{e}^{B} \mathrm{e}^{C} \mathrm{e}^{[B, C] / 2} .
$$

It is easily seen that this can be also written in the form

$$
\mathrm{e}^{B+C}=\mathrm{e}^{B / 2} \mathrm{e}^{C} \mathrm{e}^{B / 2}
$$

This identity is applicable to the position and momentum operators, since $[Q, P]=i \hbar$. Thus, we find from (2.9) and (A.3)

$$
a(\sigma, \tau)=\frac{\hbar}{2 \pi} \operatorname{tr}\left\{A(P, Q) \mathrm{e}^{-i \sigma P / 2} \mathrm{e}^{-i \tau Q} \mathrm{e}^{-i \sigma P / 2}\right\} .
$$

Within the trace we may place the last operator $\exp (-i \sigma P / 2)$ to the left of $A$ and evaluate the trace in the Schrodinger representation $|q\rangle$. Recalling that $Q|q\rangle=q \mid q>$ we have

$$
a(\sigma, \tau)=\frac{\hbar}{2 \pi} \int_{-\infty}^{+\infty} d q \mathrm{e}^{-i \tau q}<q\left|\mathrm{e}^{-i \sigma P / 2} A(P, Q) \mathrm{e}^{-i \sigma P / 2}\right| q>.
$$

Now, since $\exp (-i \sigma P / 2)|q>=| q+(\hbar \sigma / 2)>$ and $\langle q| \exp (-i \sigma P / 2)=\langle q-(\hbar \sigma / 2)|$. we have

$$
a(\sigma, \tau)=\frac{\hbar}{2 \pi} \int_{-\infty}^{+\infty} d y \mathrm{e}^{-i \tau y}<y-\frac{\hbar \sigma}{2}|A(P, Q)| y+\frac{\hbar \sigma}{2}>.
$$

Thus, from equations (2.8a) and (A.6) we have, making use of the fact that the integration over T gives $2 \pi \delta(y-q)$.

$$
A_{c}(p, q)=\hbar \int_{-\infty}^{+\infty} d \sigma \mathrm{e}^{i \sigma p}<q-\frac{\hbar \sigma}{2}|A(P, Q)| q+\frac{\hbar \sigma}{2}>.
$$

The change of variable $\hbar \sigma=x$ gives the desired equation (A.1). 